$\mathbb{T}$ periodica polytechnica

\author{
Social and Management Sciences \\ $18 / 1(2010) 39$ \\ doi: 10.3311/pp.so.2010-1.01 \\ web: http://www.pp.bme.hu/so \\ (c) Periodica Polytechnica 2010
}

RESEARCH ARTICLE

\section{Reliability based Customer Satisfaction Evaluation}

\author{
Tamás Jónás / János Kövesi
}

Received 2010-07-14

\begin{abstract}
The purpose of this article is to introduce the so-called Reliability based Customer Satisfaction Evaluation Method that is founded upon the evaluator functions using the approach of logistic-type evaluation. The scorecard based customer satisfaction measurement methods, which are widely used at Electronics Manufacturing Services (EMS) provider companies, were studied to draw conclusions on the goodness, reliability and usefulness of these traditional practices. The approach followed here was to understand the sources of uncertainties of these methods, and construct mathematical models to improve their reliability. The nature of customers' perceptions about the services they receive as functions of the scores they give through pre-agreed scorecards was mathematically modeled in evaluator functions. A method for parameter setting of evaluator functions was developed to make the functions adequately reflecting the customers' satisfaction perceptions.
\end{abstract}

\section{Keywords}

Customer satisfaction measurement and evaluation . Logistic-type evaluation - Reliability based Customer Satisfaction Evaluation Method

\section{Tamás Jónás}

Flextronics Budapest Ltd., 1183 Budapest, Hangár u. 5-37, Hungary e-mail: tamas.jonas@hu.flextronics.com

\section{János Kövesi}

Deratment of Management and Corporate Economics, BME, 1111 Budapest, Múegyetem rkp. 9., Hungary

e-mail: kovesi@mvt.bme.hu

\section{Introduction}

Understanding the customers' voice is a key contributor to success of any organization that provides services. In this paper, we analyze the nature of typical customer satisfaction measurement methods used by companies, which do not produce their own products in traditional manner, but have services, which they provide to their customers as their own products, and these customers are not the end users. Good examples for these Service Provider (SP) companies are the Electronics Manufacturing Services (EMS) providers. The sequence of ideas followed here is based on our experience we gained by investigations and studies made on the customer satisfaction practices of such companies.

A common characteristic of EMS companies is that they do not have direct contact to end users and customers of the services they provide. Typically, these produce products of Original Equipment Manufacturer (OEM) companies, and so the OEM clients are their direct customers. This difference between the EMS and OEM companies also makes differences in the attributes of their customer satisfaction measurement and evaluation practices, and there are at least two notable differences, which we need to take into consideration. One is the number of customers: in case of EMS companies, there are typically a few tens of customers, whom the services are provided to, however the original manufacturers may even have thousands, hundreds of thousands or even more direct customers. The other difference relates to the entities embodying the customers. Customers of a typical EMS company are commonly not individuals (not the end customers), but other organizations that sell goods to their markets, and so they are much closer to their end users than the companies that physically provide the services themselves.

In this article, we highlight the typical problems that occur when such service providers measure and evaluate how much their customers are satisfied with the provided services, and we introduce a novel approach of customer satisfaction evaluation. This new approach renders the handling and mitigation of problems possible through evaluator functions, and allows the organization of companies to "hear the customers' voice" better. 


\section{Measuring Customer Satisfaction}

The common way of Customer Satisfaction (CS in what follows) measurement that typical service provider companies follow is based on scorecard methods. Namely, the customers are regularly asked to give their feedback to the company using preagreed scorecards. The ultimate goal of these scorecards is to quantify the performance of the SP in areas such as quality, supply chain management, delivery accuracy, flexibility, customer communication, etc. Regardless what sort of scorecards are used, finally, an aggregated artificial number is used to characterize the level of CS for each customer. This aggregated figure is interpreted in a given preference system of the company, by this means the SP company evaluates its own performance based on the customer scorecard in its own preference system. For example, an EMS company uses a scorecard that measures the CS on a scale that goes from 0 to 100 , and every time a customer of this company is asked about his or her satisfaction, the answer is a number between 0 and 100. Based on this number and on the company's preference system, the CS may be interpreted as in the example given by Table 1

Tab. 1.

\begin{tabular}{ll}
\hline Score & Level of Customer Satisfaction \\
\hline$<20$ & Very poor \\
$\geq 20$ and $<40$ & Poor \\
$\geq 40$ and $<60$ & Meets expectations \\
$\geq 60$ and $<80$ & Above expectations \\
$\geq 80$ & Excellent \\
\hline
\end{tabular}

Certainly, the content of scorecards and the scoring criteria may vary from customer to customer, but these are always fixed, when we look at one particular customer. Let us focus only on one customer and his or her scorecard to understand the above shown measurement and evaluation. At first sight it appears that if the scorecard is well defined, and the customer has the right interpretation of scoring criteria, then the measurement is accurate and consistent, and so the evaluation reflects the real level of customer's satisfaction. Unfortunately, this is not necessarily so, as there is a number of factors that may influence the measurement and the evaluation and cause uncertainties.

\subsection{Uncertainties around the measurement}

The first thing we may observe is that the measurement based on a customer scorecard - is done by the customer, and the evaluation is made by the company. It would not be a distorting factor itself, if the measurement was really able to reflect the customer's perception of the level of received services. Is it really true that a customer is double satisfied when he or she gives 80 points compared to if he or she gives only 40 points? Thinking about this question may make us worried about the consistency of this method, although similar methods are widely used at EMS companies. The problem is that we tend to assume that a customer expresses his or her satisfaction on a linear scale (proportional scale), i.e. the score given by the customer is proportional to his or her perceived satisfaction. If it is not so, then the customer's perceived satisfaction should not be linearly evaluated. It means that the company's linear evaluation of the customer satisfaction level may be questionable, even if it seems to be absolutely rational and logical.

As we discussed earlier, the customers, we are talking about here, are not individuals even if we referred to them like they were persons. They are organizations of other companies, representatives of our customers. Whenever we use the word "customer", we mean an organization under that, the organization, who we sell our services to. On the other hand, of course, these organizations represent themselves by individuals, who may have influences on the feedbacks of their organizations, even if they try to be objective with their best intentions. Unfortunately, their subjectivity is somehow always in the scores they give, and so if we consider the scorecards as measurement systems, then the repeatability and reproducibility [1, Burdick and Borror, 2005] of these systems is questionable.

The typical role setup of a scorecard based customer satisfaction measurement and evaluation at SP companies looks so that the customer provides the scores (does the measurement) and the service provider company evaluates them. (Certainly, the evaluation results are public to the customer, who scored the performance of the service provider.) With other words, the company receives numbers, and believes that comparing these numbers to the evaluation criteria reflects how much the customer is satisfied. Therefore, the customer is not asked to give a feedback about his or her perceived satisfaction level, rather just asked to quantify the level of performance provided by the service provider company.

The above identified problems with the reliability and consistency of scorecard based CS measurements and evaluations can be summarized in the following three items.

1 CS scores are commonly measured on a proportional scale. However, in fact, the customers' perceptions about their satisfaction level are usually not proportional to the scores they give.

2 There are considerable subjective elements impacting the repeatability and reproducibility of measurement.

3 The CS measurement is commonly done by the customer using a scorecard, but the evaluation is performed by the service provider company, based on the scores received from the customer. It prohibits getting relevant information about the customer's perceived satisfaction.

\section{Evaluator functions}

Our ultimate goal is to propose a solution that can mitigate the highlighted problems with CS measurement and evaluation. We have found that a possible solution is the use of evaluator functions. 
The evaluator functions are mathematical functions that translate the scorecard based CS measurement scores to an evaluation scale. Let variable $m$ be the measured CS scores in the $\left[m_{S}, m_{E}\right]$ interval, where $m_{S}$ and $m_{E}$ are the start- and endpoint of the measurement scale. Using these notations an $E$ evaluator function assigns the $E(m) \mathrm{CS}$ value to every $m$ measured CS value, and meets the following basic criteria.

1 The $E(m)$ function is monotonously increasing, that is higher measured values correspond to higher perceived satisfaction level even if the relationship between them is not linear.

2 The range carrier of $E(m)$ is the $(0,1)$ interval.

These criteria determine just a loose frame for an evaluator function, but taking into account other experimential properties of customers' behavior and satisfaction perceptions, we can derive particular evaluator functions.

\subsection{Logistic-type evaluation}

Based on the research results derived form formal and informal interviews, and discussions with customers, we concluded on that each customer perceives a threshold on the measured CS values $(m)$, and if a particular CS value is significantly lower or higher than the threshold, then the customer is doubtlessly unsatisfied or satisfied respectively. On the other hand, if a measured CS value is close to the threshold, then the customer becomes more uncertain in the decision making situation. It suggests that the threshold that a customer perceives is rather fuzzy than sharply defined. It is also valid that the more distant the measured CS is from the threshold, the less the customer evaluation $E(m)$ changes. This attribute of the evaluation comes from the rational thought that over a certain perceived satisfaction, despite the measured CS is increasing further the customers do not feel significantly more value or utility (diminishing marginal utility). The same is valid for low values of perceived satisfaction. With other words, the slope of $E(m)$ evaluation function is low, when $E(m)$ is near $E_{L}$ or $E_{H}$, and it shows greater slope when it is more distant both from $E_{L}$ and $E_{H}$, where $E_{L}$ and $E_{H}$ are the customer's lowest and highest evaluation values. Fig. 1 illustrates the introduced properties of the $E(m)$ function.

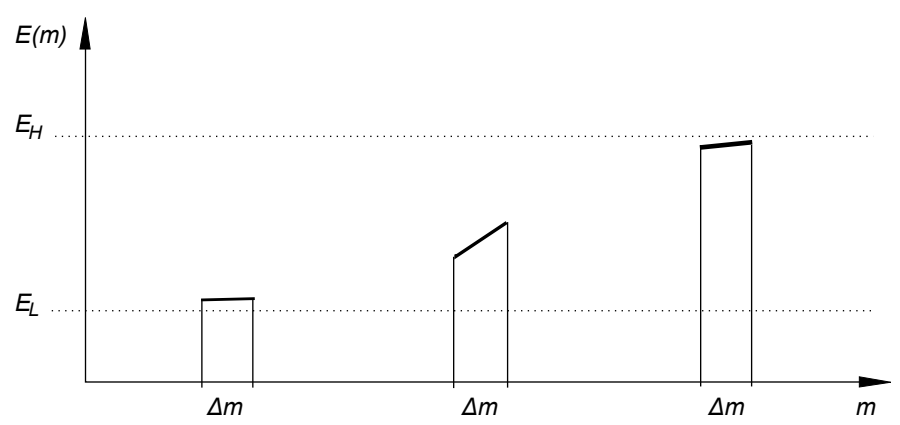

Fig. 1.

Mathematically, we can model this behavior with the

$$
\Delta E(m)=\lambda\left[E(m)-E_{L}\right]\left[E_{H}-E(m)\right] \Delta m
$$

difference equation, where $\lambda>0$ is a customer specific proportionality coefficient. Let us use the $[0,1]$ interval as evaluation scale, and set the minimum and maximum values of perceived customer satisfaction to $E_{L}=0$ and $E_{H}=1$ respectively.

We will show later, why this scale selection is practical and advantageous, and will discuss the case when $0<E_{L}<$ $E_{H}<1$. Turning into infinitesimal quantities, and applying that $E_{L}=0$ and $E_{H}=1$, Eq. (1) turns into the following differential equation.

$$
\frac{\mathrm{d} E(m)}{\mathrm{d} m}=\lambda E(m)[1-E(m)]
$$

Eq. (2) is known as logistic equation, and so we call this evaluation logistic-type evaluation. A solution for the logistic equation can be found in Lewandowski's book [2, Lewandowski, 1974]. Solving Eq. (2) results the

$$
E(m)=\frac{1}{1+e^{-\lambda m+C}}
$$

function, where $C$ is the integral constant. Setting up the $E\left(m_{0}\right)=E_{m_{0}}$ criterion (where $E_{m_{0}} \in\left(E_{L}, E_{H}\right)$ and $m_{0}$ is between $m_{S}$ and $m_{E}$ )

$$
C=\ln \frac{1-E_{m_{0}}}{E_{m_{0}}}+\lambda m_{0}
$$

and so

$$
E(m)=\frac{1}{1+\frac{1-E_{m_{0}}}{E_{m_{0}}} e^{-\lambda\left(m-m_{0}\right)}}
$$

Eq. (5) is known as logistic or sigmoid function. The logistic function - in several forms, with various parameter denotations - has a number of various applications. It was firstly introduced in 1845 , by Verhulst, who used the logistic equation to model species population growth [3. P. F. Verhulst, 1845]. Population growth models using the logistic growth can also be found in Murray's book [4, Murray, 1989]. Modis uses the function in epidemiology to describe the spreading of epidemics [5, Modis, 1992]. The logistic function can be used to model how technologies diffuse and substitute each other [6. Fisher and Pry, 1971]. Clark introduces its applications in economics [7] Clark, 1990]. The function can be used in neural networks as a threshold function [8, Mitchell, 1997], in logistic regression [9. Hosmer and Lemeshov, 1989], or in fuzzy theory as a membership function (Dombi, 1990)[10]. As the $E(m)$ function has the $\lambda, m_{0}$ and $E_{m_{0}}$ parameters, we will use the

$$
E_{\lambda, m_{0}, E_{m_{0}}}(m)=\frac{1}{1+\frac{1-E_{m_{0}}}{E_{m_{0}}} e^{-\lambda\left(m-m_{0}\right)}}
$$

notation.

\subsubsection{Properties of the $E_{\lambda, m_{0}, E_{m_{0}}}(m)$ function}

Basic properties - and the ones that are important from CS valuation point of view - of the $E_{\lambda, m_{0}, E_{m_{0}}}(m)$ function are summarized below. 


\section{- Monotony and limits}

If $\lambda$ is positive, then $E_{\lambda, m_{0}, E_{m_{0}}}(m)$ is an increasing function. It can be seen that the function converges to 1 , if $m$ tends to positive infinity, and it converges to 0 , if $m$ tends to negative infinity. (We consider here the $\lambda>0$ case only. It can be easily seen that $E_{\lambda, m_{0}, E_{m_{0}}}(m)$ is a monotonous decreasing function, if $\lambda$ is negative.)

\section{- Derivative}

Derivative of $E_{\lambda, m_{0}, E_{m_{0}}}(m)$ in the $m_{0}$ location is

$$
\frac{\mathrm{d} E_{\lambda, m_{0}, E_{m_{0}}}\left(m_{0}\right)}{\mathrm{d} m}=\lambda E_{m_{0}}\left(1-E_{m_{0}}\right)
$$

\section{- Symmetry and inflection point}

The function changes its shape from convex to concave in the

$$
\left(m_{0}+\frac{1}{\lambda} \ln \frac{1-E_{m_{0}}}{E_{m_{0}}}, \frac{1}{2}\right)
$$

point. This point is also the symmetry center of curve of the function.

\section{- Role of parameter $\lambda$}

If $E_{m_{0}}$ is fixed, then the function's (Eq. (7)) derivative in $m_{0}$ is proportional to $\lambda$, that is, it determines the slope of curve in the

$$
\left(m_{0}+\frac{1}{\lambda} \ln \frac{1-E_{m_{0}}}{E_{m_{0}}}, \frac{1}{2}\right)
$$

point. Therefore, $\lambda$ determines the sharpness of transition of the curve from 0 to 1 .

\section{- Role of parameter $m_{0}$}

The curve changes its shape in the

$$
m_{0}+\frac{1}{\lambda} \ln \frac{1-E_{m_{0}}}{E_{m_{0}}}
$$

locus. Graphically, it means that parameter $m_{0}$ determines the point where the $S$-shaped curve of function takes its place along the abscissa axis (see Fig. 2), or with other words, the

$$
\left(m_{0}+\frac{1}{\lambda} \ln \frac{1-E_{m_{0}}}{E_{m_{0}}}, \frac{1}{2}\right)
$$

point is the one, around which the curve changes from 0 to 1 .

Eq. (6) can be written in the

$$
\begin{aligned}
& E_{\lambda, m_{0}, E_{m_{0}}}(m)=\frac{1}{1+\frac{1-E_{m_{0}}}{E_{m_{0}}} e^{-\lambda\left(m-m_{0}\right)}}= \\
& \frac{1}{1+e^{-\lambda\left[m-\left(m_{0}+\frac{1}{\lambda} \ln \frac{1-E_{m_{0}}}{E_{m_{0}}}\right)\right]}}=\frac{1}{1+e^{-\frac{m-\mu}{\sigma}}}
\end{aligned}
$$

form, where

$$
\mu=m_{0}+\frac{1}{\lambda} \ln \frac{1-E_{m_{0}}}{E_{m_{0}}},
$$

$$
\sigma=\frac{1}{\lambda}
$$

The

$$
F(m ; \mu, \sigma)=\frac{1}{1+e^{-\frac{m-\mu}{\sigma}}}
$$

function is known as the cumulative distribution function of $\boldsymbol{l o}$ gistic distribution [11, Balakrishnan, 1992] $\mu$ is called location parameter, while $\sigma$ is usually referenced as scale parameter, and these naming conventions harmonize with the roles of $m_{0}$ and $\lambda$ described above. Fig. 2 shows a logistic curve with $\lambda=0.15$, $m_{0}=65, E_{m_{0}}=0.85$.

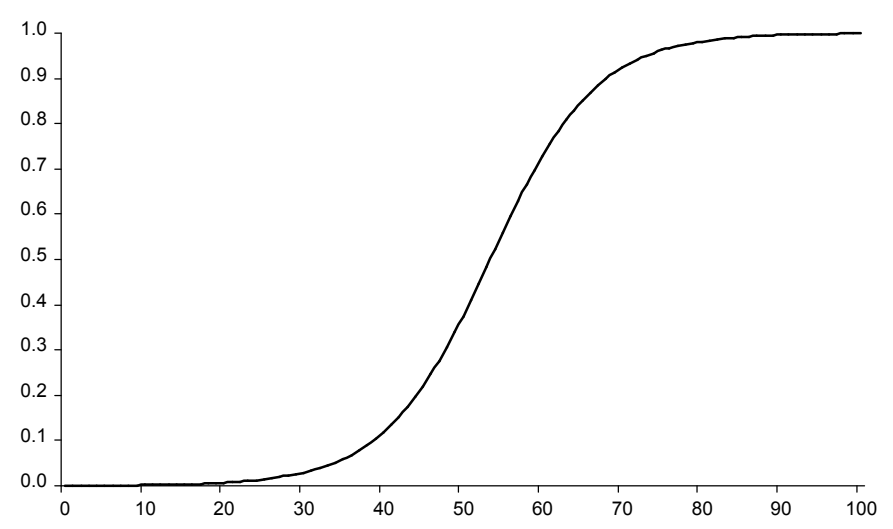

Fig. 2. Logistic curve

We have introduced why and how the logistic function can be used as an evaluator function. We found the function suitable to model the perceived CS as function of the measured CS score. On the other hand, certain properties of the sigmoid function limit its practical applicability. Although we can adjust the shape and position of function given by Eq. 6 by tuning its $\lambda$, $m_{0}$ and $E_{m_{0}}$ parameters, we cannot directly set its values in the endpoints of the $\left[m_{S}, m_{E}\right]$ interval. Therefore, we propose to use evaluator functions that are on one hand just approximants of the sigmoid functions, but on the other hand those can be parameterized with the evaluation values in endpoints of the $\left[m_{S}, m_{E}\right]$ interval.

\subsection{The $E_{\omega}(m)$ evaluator function}

Our measurement scale goes from $m_{S}$ to $m_{E}$ and function Eq. (6) has value of $E_{m_{0}}$ in the $m_{0}$ locus $\left(m_{S}<m_{0}<m_{E}\right)$. In practice, the logistic function based evaluation may not be good enough, as usually there is a need for evaluation methods that allow the customers to assign particular satisfaction values to the lowest and highest CS scores on the measurement scale. A customer may not be completely unsatisfied (or satisfied), if the CS score is minimal (or maximal). This suggests that the evaluator functions need to have parameters that render the customers to set their minimum and maximum satisfaction values to the minimal and maximal CS scores. 
It can be proven that the following

$$
\begin{aligned}
& E_{\omega, m_{S}, m_{E}, m_{0}, E_{m_{0}}}(m)= \\
& =\frac{\left(\frac{m-m_{S}}{m_{E}-m_{S}}\right)^{\omega}}{\left(\frac{m-m_{S}}{m_{E}-m_{S}}\right)^{\omega}+\frac{1-E_{m_{0}}}{E_{m_{0}}}\left[\frac{m_{0}-m_{S}}{m_{E}-m_{0}}\left(1-\frac{m-m_{S}}{m_{E}-m_{S}}\right)\right]^{\omega}}
\end{aligned}
$$

is a good approximant of the $E_{\lambda, m_{0}, E_{m_{0}}}(m)$ function, if

$$
\omega=\frac{\lambda\left(m_{E}-m_{0}\right)\left(m_{0}-m_{S}\right)}{m_{E}-m_{S}} .
$$

Eq.(10) is a linearly transformed version of Dombi's $\kappa$ function that he introduced in the fuzzy theory as a membership function [10, Dombi, 1990].

$E_{\omega, m_{S}, m_{E}, m_{0}, E_{m_{0}}}\left(m_{0}\right)=E_{m_{0}}$, and the $\omega=$ $\lambda\left(m_{E}-m_{0}\right)\left(m_{0}-m_{S}\right) /\left(m_{E}-m_{S}\right) \quad$ criterion ensures that $E_{\lambda, m_{0}, E_{m_{0}}}(m)$ and $E_{\omega, m_{S}, m_{E}, m_{0}, E_{m_{0}}}(m)$ have the same slope in the $m_{0}$ location.

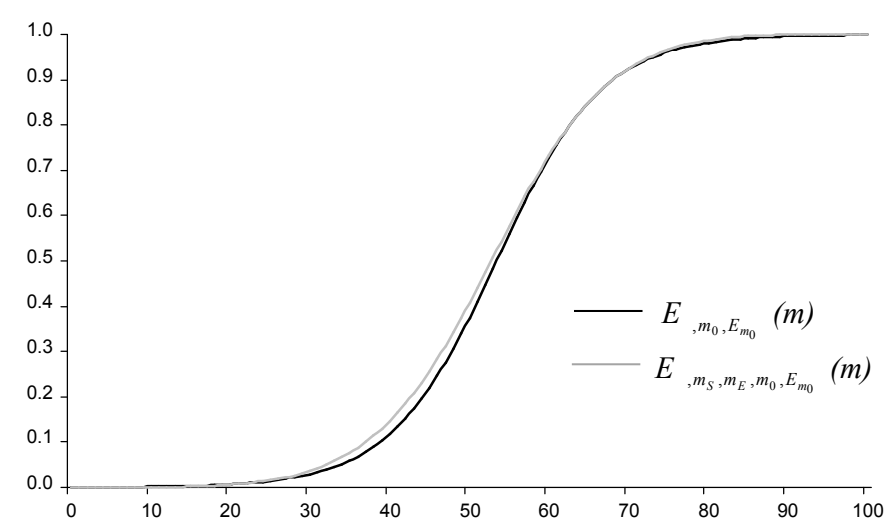

Fig. 3. $E_{\lambda, m_{0}, E_{m_{0}}}(m)$ and $E_{\omega, m_{S}, m_{E}, m_{0}, E_{m_{0}}}(m)$

For example, Fig. 3 shows the graphs of $E_{\lambda, m_{0}, E_{m_{0}}}(m)$ and $E_{\omega, m_{S}, m_{E}, m_{0}, E_{m_{0}}}(m)$ functions with $\lambda=0.15, m_{S}=0, m_{E}=$ $100, m_{0}=65, E_{m_{0}}=0.85$ and $\omega=3.4125$ parameters. In this example the maximum difference between the two functions is 0.0371 .

$E_{\omega, m_{S}, m_{E}, m_{0}, E_{m_{0}}}\left(m_{S}\right)=0$, and $E_{\omega, m_{S}, m_{E}, m_{0}, E_{m_{0}}}\left(m_{E}\right)=1$, that is the $E_{\omega, m_{S}, m_{E}, m_{0}, E_{m_{0}}}(m)$ function assigns the 0 and 1 satisfaction values to the lowest and highest CS scores respectively. As we touched on earlier, we need to have the option to set the satisfaction values in the endpoints of the measurement scale. For this purpose, we can create Eq. (12) that is a generalized version of the $E_{\omega, m_{S}, m_{E}, m_{0}, E_{m_{0}}}(m)$ function.

$$
\begin{aligned}
& E_{\omega, m_{S}, m_{E}, m_{0}, E_{m_{0}}, E_{L}, E_{H}}(m)= \\
& =E_{L}+\left(E_{H}-E_{L}\right) \frac{\left(\frac{m-m_{S}}{m_{E}-m_{S}}\right)^{\omega}}{\left(\frac{m-m_{S}}{m_{E}-m_{S}}\right)^{\omega}+\frac{E_{H}-E_{m_{0}}}{E_{m_{0}}-E_{L}}\left[\frac{m_{0}-m_{S}}{m^{-} E^{-m_{0}}}\left(1-\frac{m-m_{S}}{m_{E}-m_{S}}\right)\right]^{\omega}}
\end{aligned}
$$

The $E_{\omega, m_{S}, m_{E}, m_{0}, E_{m_{0}}, E_{L}, E_{H}}(m)$ evaluator function has seven parameters: $\omega, m_{S}, m_{E}, m_{0}, E_{m_{0}}, E_{L}$ and $E_{H}$. The $m_{S}, m_{E}$, $E_{L}, E_{H}$ parameters are the window parameters as these determine a rectangle shaped area in the orthogonal system of coordinates. The bottom side of this rectangle is the measurement scale, while its left side is the evaluation scale. Parameter $\omega$ has the same role as for Eq. 10, and $\lambda$ for the logistic function, that is $\omega$ determines the slope of evaluator function in the $m_{0}$ location. From this point onwards, we will use the simplified $E_{\omega}(m)$ notation instead of the $E_{\omega, m_{S}, m_{E}, m_{0}, E_{m_{0}}, E_{L}, E_{H}}(m)$ long form, and refer to Eq. 12 as $E_{\omega}(m)$ evaluator function. The advantage of the $E_{\omega}(m)$ function is that it allows us to assign the $E_{L}$ and $E_{H}$ satisfaction values to the $m_{S}$ and $m_{E}$ endpoints of the CS measurement scale. A version of the $E_{\omega}(m)$ function, with another parameter set can also be used for valuation of intellectual capital of corporate organizations [12, Jónás et al.].

\subsection{Practical use of the $E_{\omega}(m)$ function}

We derived the $E_{\omega}(m)$ function as an approximant of the sigmoid function, and interpreted the roles of its parameters. As we discussed earlier, one of the problems with the commonly used CS evaluation is that the measurement is done by the customer (based on a scorecard) and the evaluation is done by the service provider (EMS company), that is these two process steps are separate. This separation in itself would not cause any problem, if the evaluation performed by the service provider company could adequately reflect the customer's perception. One of our findings is that in practice, there is a disconnection between customer's and service provider's evaluations.

Now, we have a mathematical tool that the customer can use to evaluate his or her satisfaction using the CS scorecard. In order to really make it possible, the customer needs to set the parameters of the evaluator function. The key thing is that the customer calibrates the evaluator function, that is sets its parameters so that the function reflects customer's satisfaction perception of the measured CS scores.

Here we describe the method how to use the $E_{\omega}(m)$ evaluator function for customer satisfaction evaluation. We call this method Reliability based Customer Satisfaction Evaluation Method. The $E_{\omega}(m)$ function can be considered as one that reevaluates the CS scores given by a customer and by this means it allows the customer to express his/her perceived satisfaction better, even if the service provider uses the same, standardized and common scorecard based system to measure the CS scores of its different customers. Finally, this approach increases the reliability of the satisfaction evaluation.

\subsubsection{Step 1}

At first we ask the customer to measure his/her satsifaction based on a common scorecard system that we use for all the customers.

\subsubsection{Step 2}

The customer needs to set the window parameters for the $E_{\omega}(m)$ function. These determine the domain of variability (the $\left[m_{S}, m_{E}\right]$ interval) and the lowest $\left(E_{L}\right)$ and highest $\left(E_{H}\right)$ satisfaction values of the $[0,1]$ evaluation scale. 


\subsubsection{Step 3}

There are three further parameters, the $m_{0}, E_{m_{0}}$ and $\omega$ that need to be specified to unambiguously determine the evaluator function. For this purpose we need to ask the customer to specify two satisfaction levels on the evaluation scale in the $\left(E_{L}, E_{H}\right)$ interval that he/she assigns to two arbitrary chosen (but different both from $m_{S}$ and $m_{E}$ ) points of the original CS measurement scale. By this means the customer specifies two (measurement value, evaluation value) pairs. Any one of these two pairs can be directly used as the $\left(m_{0}, E_{m_{0}}\right)$ pair, and so one point of $E_{\omega}(m)$ is explicitly given. In practice, we recommend to select $m_{0}$ as the midpoint of the measurement scale since this - as the half of the maximum reachable score - is a good characteristic point of it.

\subsubsection{Step 4}

Let $\left(m_{a}, E_{m_{a}}\right)$ note the other arbitrary chosen (measurement value, evaluation value) pair. As $\left(m_{a}, E_{m_{a}}\right)$ is a point of the $E_{\omega}(m)$ curve, the

$$
\begin{aligned}
E_{m_{a}} & =E_{L}+\left(E_{H}-E_{L}\right) \cdot \\
\cdot & \frac{\left(\frac{m_{a}-m_{S}}{m_{E}-m_{S}}\right)^{\omega}}{\left(\frac{m_{a}-m_{S}}{m_{E}-m_{S}}\right)^{\omega}+\frac{E_{H}-E_{m_{0}}}{E_{m_{0}}-E_{L}}\left[\frac{m_{0}-m_{S}}{m_{E}-m_{0}}\left(1-\frac{m_{a}-m_{S}}{m_{E}-m_{S}}\right)\right]^{\omega}}
\end{aligned}
$$

equation needs to be met. From this equation parameter $\omega$ can be calculated as

$$
\omega=\frac{\ln \left(\frac{E_{H}-E_{m_{a}}}{E_{m_{a}}-E_{L}} \frac{E_{m_{0}}-E_{L}}{E_{H}-E_{m_{0}}}\right)}{\ln \left(\frac{m_{E}-m_{a}}{m_{a}-m_{S}} \frac{m_{0}-m_{S}}{m_{E}-m_{0}}\right)}
$$

Which point of the measurement scale is worth to be chosen for $m_{a}$ ? Based on the interviews we had with customers, we may state that each of them has a kind of a threshold value for the measured CS score as we referred to that earlier. Certainly, these threshold figures vary from customer to customer, and basically that is why the standardized scorecard based measurement is limited in its capability to express the customer satisfaction appropriately. The method we introduced and discussed so far resolves this problem through customer specific evaluator functions by allowing the customers to assign their values of perceived satisfaction to the scores measured by a standardized scorecard method. Hence, we recommend setting $m_{a}$ as the customer specific threshold value for the measured CS score for each customer.

\subsubsection{An example}

In this example, the interviewed customer has agreed with the assumption that his perceived satisfaction has a logistic-type nature as we described in the section about the logistic-type evaluation. The customer satisfaction scorecard has a scale that goes from 0 to 100, while the minimum and maximum values on the vertical axis are 0.1 and 1 , that is the window parameters are $m_{S}=0, m_{E}=100, E_{L}=0.1, E_{H}=1$. The customer have chosen the $\left(m_{0}=50, E_{m_{0}}=0.3\right)$ and $\left(m_{a}=90, E_{m_{a}}=0.95\right)$ points to calibrate the evaluator function. It means that CS score of 50 has value of 0.3 in customer's preference system, as well as customer assigns 0.95 value to score of 90 . Based on these settings $\omega$ can be calculated as

$\omega=\frac{\ln \left(\frac{E_{H}-E_{m_{a}}}{E_{m_{a}}-E_{L}} \frac{E_{m_{0}}-E_{L}}{E_{H}-E_{m_{0}}}\right)}{\ln \left(\frac{m_{E}-m_{a}}{m_{a}-m_{S}} \frac{m_{0}-m_{S}}{m_{E}-m_{0}}\right)}=\frac{\ln \left(\frac{1-0.95}{0.95-0.1} \frac{0.3-0.1}{1-0.3}\right)}{\ln \left(\frac{100-90}{90-0} \frac{50-0}{100-50}\right)}=1.8596$.

We must keep in mind that the $E_{\omega}(m)$ function is an approximant of the sigmoid function, and so it is important to see that the calculations above are valid only in the case, when the nature of customer's perceived satisfaction follows the logistic equation. Certainly, other evaluator functions can be applicable too however, our finding is that the logistic-type evaluation is one that models the customer's behavior well.

\subsection{Customer's voice as an input for performance goal set- ting}

If a service provider company uses the $E_{\omega}(m)$ function to understand the customer's perception, it allows the company to set the performance goals in terms of the customer's perceived satisfaction instead of setting these goals in terms of the customer satisfaction score, whose consistency is questionable. The company can ask the customer to specify the $\left(m_{0}, E_{m_{0}}\right)$ and $\left(m_{a}, E_{m_{a}}\right)$ pairs and an $E_{\text {Target }}$ level of the evaluated CS that he or she deems good enough to be used as a target. Using the inverse of $E_{\omega}(m)$ function, the company can translate $E_{\text {Target }}$ to a CS score $\left(E_{\omega}(m)\right.$ is invertible as it is strongly monotonic). In practice, the CS score, for the company, is a blended performance metric with clear definition. It means that translating the customer perception to this metric can help the management identifying the actions required to achieve the necessary level of CS score, which at the same time corresponds to the "real" customer satisfaction.

\subsection{Utility point of view}

We discussed so far that use of the introduced CS evaluator functions is a possible way of getting information about our performance, if we are in the role of a service provider company. The evaluated customer satisfaction represents the level of performance that our customers perceive. It means that from the company's point of view the customer satisfaction is a measure of performance, while from a customer's perspective, the level of satisfaction is the measure of utility of services that the company provides. Therefore, the CS evaluator function can be interpreted as utility functions as well.

\section{Summary}

In this article, we studied and analyzed the characteristics of typical customer satisfaction measurements and evaluations used by EMS companies, and concluded on what factors made 
the common methods uncertain. Our finding is that the scorecard based measurement methods are uncertain, and their uncertainty is due to their nature.

As a new approach, our recommendation is to use CS evaluator functions that translate the scorecard based CS measurement scores to an evaluation scale. This transformation allows the customers to express their perceptions of the level of satisfaction resulted by the services they receive.

We discussed the Reliability based Customer Satisfaction Method that is founded on the logistic-type evaluation and pointed out why the sigmoid function is a suitable model to link the measured scores to the customer perceived satisfaction. We introduced the $E_{\omega}(m)$ function - as an approximant of the logistic function - to surmount the practical limitations of sigmoid function. As the $E_{\omega}(m)$ function can be customer specifically calibrated based on inputs of a customer, our method enables a service provider company to understand the satisfaction perception of this customer better.

Practical cases have proven that our research results are applicable in practice, and the $E_{\omega}(m)$ function is adequate for evaluation purposes. Although our research was limited to EMS companies, it can also be applied by any service provider company. One of our further plans is to investigate how the methods found can be applied to other types of companies. We also plan to do further research on the aggregation of customer satisfaction evaluation in business environments, where the number of customers is large.

\section{References}

1 Burdick R, Borror C, Montgomery D, Design and Analysis of Gauge RER Studies, Series on Statistics and Applied Probability, SIAM, Philadelphia, 2005, ISBN 0898715881.

2 Lewandowski R, Prognose- und Informationssysteme und ihre Anwendungen, Walter de Gruyter, Berlin, 1974.

3 Verhulst $\mathbf{P}$, Recherches mathématiques sur la loi d'accroissement de la population., Nouveaux Mémoirs de l'Académie Royale des Sciences et des Belles-Lettres de Bruxelles, 18, (1848). Retrieved in 2009.

4 Murray J, Mathematical Biology, Springer, New York, 1989.

5 Modis T, Predictions: Society's Telltale Signature Reveals the Past and Forecasts the Future, Simon \& Schuster, New York, 1992.

6 Fisher J, Pry R, A Simple Substitution Model of Technological Change, Technological Forecasting \& Social Change, 3(1), (1971), 75-88, DOI 10.1016/S0040-1625(71)80005-7.

7 Clark C, Mathematical Bioeconomics: The Optimal Management of Renewable Resources, Wiley Interscience, New York, 1990.

8 Mitchell T, Machine Learning, WCB-McGraw-Hill, Boston, 1997.

9 Hosmer D, Lemeshov S, Applied Logistic regression, Wiley \& Sons, New York, 1989.

10 Dombi J, Membership function as an evaluation, Fuzzy Sets and Systems, 35(1), (1990), 1, DOI 10.1016/0165-0114(90)90014-W.

11 Balakrishnan N, Handbook of the Logistic Distribution, Marcel Dekker, New York, 1992.

12 Jónás T, Kövesi J, Tóth $\mathbf{Z}, A z$ intellektuális tôke mérésének és értékelésének egyes kérdései, Vezetéstudomány, 15, (2009), 24-29. 